\title{
ADDENDUM
}

\section{A bargaining game analysis of international climate negotiations}

Rory Smead, Ronald L. Sandler, Patrick Forber and John Basl

Nature Climate Change 4, 442-445 (2014); published online 11 May 2014; addendum published after print 8 August 2014.

In the Supplementary Information file for this Letter, the authors have added a section titled "Description of simulation procedure", which provides a detailed account of the primary simulation procedure used in the study. This section is intended to allow others to directly replicate the results, and contains some detail and accompanying explanation omitted from the original version. The revised version also includes notational corrections to the proof of Proposition 1. 